\title{
THE SINE AND COSINE RULES FOR PURE TRIANGLES ON THE DUAL LORENTZIAN UNIT SPHERE $\widetilde{S}_{1}^{2}$
}

\author{
M. Kazaz \\ Department of Mathematics, Faculty of Science, University of Celal Bayar, \\ Muradiye Campus,45047, Manisa, Turkey.m_kazaz@hotmail.com
}

\begin{abstract}
In this work, we proved the sine and cosine rules for a spherical pure triangle on the dual Lorentzian unit sphere $\widetilde{S}_{1}^{2}$ in the dual Lorentzian space $D_{1}^{3}$.
\end{abstract}

Keywords- Dual Lorentzian space, dual unit sphere, spacelike and timelike vectors.

\section{INTRODUCTION}

Dual numbers had been introduced by W. K. Clifford (1845-1879) as a tool for his geometrical investigations. After him, E. Study used dual numbers and dual vectors in his research on line geometry and kinematics [6]. He devoted special attention to the representation of directed line by dual vectors and defined the mapping that is said with his name; he proved that there exists one to one correspondence between the points of the dual unit sphere $\widetilde{S}^{2}$ and the directed lines of the Euclidean 3-space $I R^{3}$ (E. Study's mapping ).

In [7], by taking the Minkowski 3-space $I R_{1}^{3}$ instead of $I R^{3}$, Uğurlu and Çalışkan gave a correspondence of E. Study's mapping as follows: There exist one to one correspondence between the dual spacelike unit vectors of dual Lorentzian unit sphere $\widetilde{S}_{1}^{2}$ in the dual Lorentzian space $D_{1}^{3}$ and directed spacelike lines of the Minkowski 3-space $I R_{1}^{3}$.

In plane Lorentzian geometry it is studied points, timelike, spacelike, and ligtlike lines, triangles, etc [3]. On the Lorentzian sphere, there are points, but there are no straight lines, at least not in the usual sense. However, straight timelike, spacelike and lightlike lines in the Lorentzian plane are characterized by the fact that they are the shortest paths between points. The curves on the Lorentzian sphere with the same property are timelike, spacelike and lightlike circles. Thus it is natural to use these circles as replacements for lines.

The formulas for the sine and cosine rules are given for Euclidean plane $E^{2}$ [2, 4], Lorentzian plane $I R_{1}^{2}$ [3], Euclidean sphere $S^{2}[2,4]$, hyperbolic sphere $H_{0}^{2}[2,5]$, and dual Euclidean sphere $\widetilde{S}^{2}[8]$.

The aim of this work is to prove the sine and cosine rules for a spherical pure triangle on the dual Lorentzian unit sphere $\widetilde{S}_{1}^{2}$.

\section{DUAL NUMBERS AND DUAL LORENTZIAN VECTORS}

In this section we give a brief summary of the theory of dual numbers and dual Lorentzian vectors.

Let $I R_{1}^{3}$ be a 3-dimensional Minkowski space over the field of real numbers $I R$ with the Lorentzian inner product $<,>$ given by 


$$
<a, b>=a_{1} b_{1}+a_{2} b_{2}-a_{3} b_{3},
$$

where $a=\left(a_{1}, a_{2}, a_{3}\right)$ and $b=\left(b_{1}, b_{2}, b_{3}\right) \in I R^{3}$.

A vector $a=\left(a_{1}, a_{2}, a_{3}\right)$ of $I R_{1}^{3}$ is said to be timelike if $<a, a><0$, spacelike if $<a, a>>0$, and lightlike (or null) if $<a, a>=0$. The norm of a vector $a$ is defined by $|a|=\sqrt{|<a, a\rangle \mid}$. Now let $a=\left(a_{1}, a_{2}, a_{3}\right)$ and $b=\left(b_{1}, b_{2}, b_{3}\right)$ be two vectors in $I R_{1}^{3}$, then the Lorentzian cross product is given by

$$
a \times b=\left(a_{3} b_{2}-a_{2} b_{3}, a_{1} b_{3}-a_{3} b_{1}, a_{1} b_{2}-a_{2} b_{1}\right) .
$$

A dual number has the form $\hat{\lambda}:=\lambda+\varepsilon \lambda^{*}$, where $\lambda$ and $\lambda^{*}$ are real numbers and $\varepsilon$ stands for the dual unit which is subject to the rules:

$$
\varepsilon \neq 0, \varepsilon^{2}=0,0 \varepsilon=\varepsilon 0=0,1 \varepsilon=\varepsilon 1=\varepsilon .
$$

We denote the set of dual numbers by $D$ :

$$
D=\left\{\hat{\lambda}=\lambda+\varepsilon \lambda^{*}: \lambda, \lambda^{*} \in I R, \varepsilon^{2}=0\right\} .
$$

Equality, addition and multiplication are defined in $D$ by

$$
\begin{gathered}
\lambda+\varepsilon \lambda^{*}=\beta+\varepsilon \beta^{*} \text { if and only if } \lambda=\beta \text { and } \lambda^{*}=\beta^{*}, \\
\left(\lambda+\varepsilon \lambda^{*}\right)+\left(\beta+\varepsilon \beta^{*}\right)=(\lambda+\beta)+\varepsilon\left(\lambda^{*}+\beta^{*}\right),
\end{gathered}
$$

and

$$
\left(\lambda+\varepsilon \lambda^{*}\right)\left(\beta+\varepsilon \beta^{*}\right)=\lambda \beta+\varepsilon\left(\lambda \beta^{*}+\lambda^{*} \beta\right),
$$

respectively. Then it is easy to show that $(D,+,$.$) is a commutative ring with unity.$ The numbers $\varepsilon \lambda^{*}$ are divisors of 0 . We note that if $\lambda$ and $\beta$ are two nonzero elements of a ring $R$ such that $\lambda \beta=0$, then $\lambda$ and $\beta$ are divisors of 0 ( or 0 divisors ) by

Moreover, if $\hat{\lambda}=\lambda+\varepsilon \lambda^{*}, \hat{\beta}=\beta+\varepsilon \beta^{*} \in D$ with $\beta \neq 0$ then the division is given

$$
\frac{\hat{\lambda}}{\hat{\beta}}=\frac{\lambda+\varepsilon \lambda^{*}}{\beta+\varepsilon \beta^{*}}=\frac{\lambda}{\beta}+\varepsilon\left(\frac{\lambda^{*}}{\beta}-\frac{\lambda \beta^{*}}{\beta^{2}}\right) .
$$

Now let $f$ be a differentiable function. Then the Maclaurin series generated by $f$ is

$$
f(\hat{x})=f\left(x+\varepsilon x^{*}\right)=f(x)+\varepsilon x^{*} f^{\prime}(x),
$$

where $f^{\prime}(x)$ is the derivative of $f$. Then we have

$$
\begin{aligned}
\sin h\left(x+\varepsilon x^{*}\right) & =\sin h x+\varepsilon x^{*} \cos h x, \\
\cos h\left(x+\varepsilon x^{*}\right) & =\cos h x+\varepsilon x^{*} \sin h x, \\
\cos \left(x+\varepsilon x^{*}\right) & =\cos x-\varepsilon x^{*} \sin x, \\
\sin \left(x+\varepsilon x^{*}\right) & =\sin x+\varepsilon x^{*} \cos x, \\
\sqrt{x+\varepsilon x^{*}} & =\sqrt{x}+\varepsilon \frac{x^{*}}{2 \sqrt{x}}, \quad(x>0) .
\end{aligned}
$$

The norm of $|\hat{x}|$ of a dual number $\hat{x}=x+\varepsilon x^{*}$ defined by

$$
|\hat{x}|=\left|x+\varepsilon x^{*}\right|=\sqrt{\hat{x}^{2}}=\sqrt{x^{2}+2 \varepsilon x x^{*}} .
$$


Then the formula (5) allows us to write

Thus we have

$$
|\hat{x}|=\left|x+\varepsilon x^{*}\right|=|x|+\varepsilon x^{*} \frac{x}{|x|} \quad(x \neq 0) .
$$

$$
|\hat{x}|= \begin{cases}\hat{x}, & \text { if } \hat{x}>0 \\ 0, & \text { if } \hat{x}=0 \\ -\hat{x}, & \text { if } \quad \hat{x}<0\end{cases}
$$

Let $D^{3}$ be the set of all triples of dual numbers, i.e.

$$
D^{3}=\left\{\widetilde{a}=\left(a_{1}, a_{2}, a_{3}\right) \mid a_{i} \in D, \quad i=1,2,3\right\} .
$$

The elements of $D^{3}$ are called as dual vectors. A dual vector $\tilde{a}$ may be expressed in the form $\tilde{a}=a+\varepsilon a^{*}$, where $a$ and $a^{*}$ are the vectors of $I R^{3}$.

Now let $\widetilde{a}=a+\varepsilon a^{*}, \widetilde{b}=b+\varepsilon b^{*} \in D^{3}$ and $\hat{\lambda}=\lambda_{1}+\varepsilon \lambda_{1}^{*} \in D$ then we define

$$
\begin{aligned}
& \tilde{a}+\tilde{b}=a+b+\varepsilon\left(a^{*}+b^{*}\right), \\
& \hat{\lambda} \tilde{a}=\lambda_{1} a+\varepsilon\left(\lambda_{1} a^{*}+\lambda_{1}^{*} a\right) .
\end{aligned}
$$

Then $D^{3}$ becomes a unitary $D$-module with these operations. It is called $D$-module or dual space (see, [1] for real Lorentzian concepts).

The Lorentzian inner product of two dual vectors $\widetilde{a}=a+\varepsilon a^{*}, \widetilde{b}=b+\varepsilon b^{*}$ is defined by

$$
<\tilde{a}, \tilde{b}>=<a, b>+\varepsilon\left(<a, b^{*}>+<a^{*}, b>\right),
$$

where $\langle a, b\rangle$ is the Lorentzian inner product of the vectors $a$ and $b$ in the Minkowski 3-space $I R_{1}^{3}[7]$.

A dual vector $\tilde{a}=a+\varepsilon a^{*}$ is said to be timelike if $<\tilde{a}, \widetilde{a}><0$, spacelike if $<\tilde{a}, \tilde{a}>>0$, and lightlike (or null) if $<\tilde{a}, \tilde{a}>=0$.

The set of dual timelike, spacelike and lightlike vectors is called dual Lorentzian space and it is denoted by $D_{1}^{3}$, i.e.

$$
D_{1}^{3}=\left\{\tilde{a}=a+\varepsilon a^{*} \mid a, a^{*} \in I R_{1}^{3}\right\} .
$$

The Lorentzian cross product of dual vectors $\widetilde{a}$ and $\widetilde{b} \in D_{1}^{3}$ is defined by

$$
\tilde{a} \times \tilde{b}=a \times b+\varepsilon\left(a^{*} \times b+a \times b^{*}\right),
$$

where $a \times b$ is the Lorentzian cross product in $I R_{1}^{3}$.

Lemma 2.1. Let $\widetilde{a}, \widetilde{b}, \widetilde{c}, \widetilde{d} \in D_{1}^{3}$. Then we have

$$
\begin{aligned}
& <\tilde{a} \times \tilde{b}, \widetilde{c}>=-\operatorname{det}(\tilde{a}, \tilde{b}, \widetilde{c}) \\
& \tilde{a} \times \tilde{b}=-\tilde{b} \times \widetilde{a}, \\
& (\tilde{a} \times \tilde{b}) \times \widetilde{c}=-<\widetilde{a}, \widetilde{c}>\widetilde{b}+<\widetilde{b}, \widetilde{c}>\widetilde{a}, \\
& <\tilde{a} \times \tilde{b}, \widetilde{c} \times \tilde{d}>=-<\widetilde{a}, \widetilde{c}><\tilde{b}, \tilde{d}>+<\widetilde{a}, \tilde{d}><\widetilde{b}, \widetilde{c}>\text {, } \\
& <\tilde{a} \times \tilde{b}, \tilde{a}>=0 ; \text { and }<\tilde{a} \times \tilde{b}, \tilde{b}>=0 .
\end{aligned}
$$


Let $\tilde{a}=a+\varepsilon a^{*} \in D_{1}^{3}$. Then $\tilde{a}$ is said to be dual spacelike unit vector if the vectors $a$ and $a^{*}$ satisfy the following equations

$$
<a, a>=1,<a, a^{*}>=0 .
$$

The set of all dual spacelike unit vectors is called the dual Lorentzian unit sphere, and is denoted by $\widetilde{S}_{1}^{2}$ (for more details, see [7, 8]).

Theorem 2.2 (E. Study's Mapping) The dual spacelike unit vectors of the dual Lorentzian unit sphere ${\widetilde{S_{1}}}_{1}^{2}$ are in one to one correspondence with the directed spacelike lines of the Minkowski 3-space $I R_{1}^{3} \quad$ [7].

\section{THE SINE AND COSINE RULES FOR DUAL LORENTZIA SPHERICAL TIMELIKE PURE TRIANGLES}

In this section we prove the sine and cosine rules for dual Lorentzian spherical pure triangles.

Let $\widetilde{A}, \widetilde{B}$ and $\widetilde{C}$ be three points on dual Lorentzian unit sphere $\widetilde{S}_{1}^{2}$ given by the linearly independent dual spacelike unit vectors $\widetilde{a}=a+\varepsilon a^{*}, \widetilde{b}=b+\varepsilon b^{*}$ and $\widetilde{c}=c+\varepsilon c^{*}$, respectively. These points together with the great-timelike-circle-arcs $\widetilde{A} \widetilde{B}, \widetilde{B} \widetilde{C}, \widetilde{C} \widetilde{A}$ form a dual Lorentzian spherical pure triangle $\widetilde{A} \widetilde{B} \widetilde{C}$, see Figure 3.1.

We will suppose that $\operatorname{det}(a, b, c)>0$. We denote the dual spacelike unit vectors with the same sense as $\tilde{b} \times \widetilde{c}, \widetilde{c} \times \widetilde{a}$ and $\widetilde{a} \times \widetilde{b}$ by $\tilde{n}_{a}, \tilde{n}_{b}$ and $\tilde{n}_{c}$, respectively. The side $\tilde{a}$ of $\widetilde{A} \widetilde{B} \widetilde{C}$ is defined as the dual central angle for which

$$
<\widetilde{b}, \widetilde{c}>=-\cosh \widetilde{a}, \widetilde{b} \times \widetilde{c}=\tilde{n}_{a} \sinh \tilde{a} .
$$

It can be given similar definitions for the other sides $\widetilde{b}$ and $\widetilde{c}$ of $\widetilde{A} \widetilde{B} \widetilde{C}$. Thus we have

$$
\begin{aligned}
& <\widetilde{a}, \widetilde{b}>=-\cosh \widetilde{c}, \tilde{a} \times \widetilde{b}=\tilde{n}_{c} \sinh \widetilde{c} \\
& <\widetilde{c}, \widetilde{a}>=-\cosh \widetilde{b}, \widetilde{c} \times \widetilde{a}=\widetilde{n}_{b} \sinh \tilde{b}=-\widetilde{a} \times \widetilde{c} .
\end{aligned}
$$

Since we can write $\widetilde{n}_{a}=n_{a}+\varepsilon n_{a}^{*}$, it is obvious that $n_{a}$ is the real spacelike unit vector having the same sense as $b \times c$. If $\widetilde{a}=a+\varepsilon a^{*}$, we have $\sinh a>0$. This means that $|\sinh \widetilde{a}|=\sinh \widetilde{a}$, and similarly $|\sinh \tilde{b}|=\sinh \tilde{b},|\sinh \widetilde{c}|=\sinh \widetilde{c}$. It is obvious that $\tilde{a}, \tilde{b}$ and $\widetilde{c}$ are the dual spacelike unit vectors having the same sense as $\widetilde{n}_{b} \times \widetilde{n}_{c}, \widetilde{n}_{c} \times \widetilde{n}_{a}$ and $\tilde{n}_{a} \times \tilde{n}_{b}$, respectively. given by

The dual spacelike angle $\widetilde{\alpha}$ of $\widetilde{A} \widetilde{B} \widetilde{C}$ is defined as the dual hyperbolic angle

$$
<\tilde{n}_{b}, \tilde{n}_{c}>=\cosh \tilde{\alpha}, \tilde{n}_{b} \times \tilde{n}_{c}=\tilde{a} \sinh \tilde{\alpha} .
$$

The dual spacelike angles $\widetilde{\beta}$ and $\widetilde{\gamma}$ of $\widetilde{A} \widetilde{B} \widetilde{C}$ can be defined similarly. 


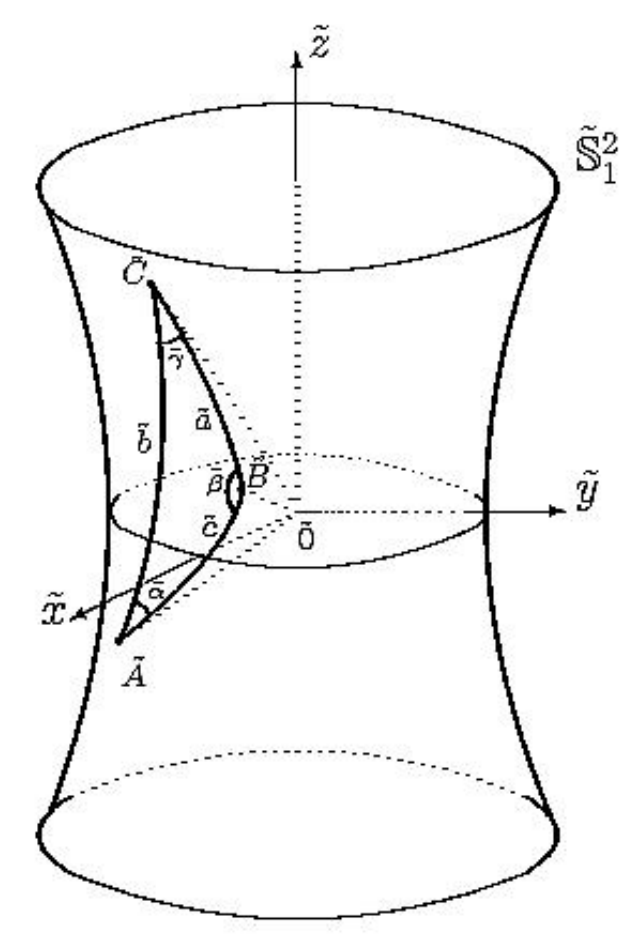

Figure 3.1: Dual Lorentzian Spherical Pure Triangle.

Lemma 3.1. (The Dual Lorentzian Cosine Rule I) Let $\widetilde{A} \widetilde{B} \widetilde{C}$ be a Lorentzian pure triangle on the dual Lorentzian unit sphere $\widetilde{S}_{1}^{2}$. Then the dual Lorentzian cosine rule I is given by

$$
\cosh \tilde{\alpha}=\frac{\cosh \tilde{b} \cosh \tilde{c}+\cosh \tilde{a}}{-\sinh \tilde{b} \sinh \widetilde{c}} .
$$

Proof: We shall calculate $<\widetilde{a} \times \widetilde{c}, \widetilde{a} \times \tilde{b}>$ in two ways. First by the formula (6), we obtain

$$
\begin{aligned}
<\widetilde{a} \times \widetilde{c}, \widetilde{a} \times \widetilde{b}> & =<-\widetilde{n}_{b} \sinh \widetilde{b}, \tilde{n}_{c} \sinh \widetilde{c}> \\
& =-<\widetilde{n}_{b}, \widetilde{n}_{c}>\sinh \tilde{b} \sinh \widetilde{c} \\
& =-\cosh \widetilde{\alpha} \sinh \tilde{b} \sinh \widetilde{c} .
\end{aligned}
$$

Secondly, by Lemma 2.1,

$$
\begin{aligned}
& <\widetilde{a} \times \widetilde{c}, \widetilde{a} \times \tilde{b}>=-<\widetilde{a}, \widetilde{a}><\widetilde{c}, \widetilde{b}>+<\widetilde{a}, \widetilde{b}><\widetilde{c}, \widetilde{a}>, \\
& =-<\tilde{c}, \tilde{b}>+<\tilde{a}, \tilde{b}><\widetilde{c}, \tilde{a}>, \\
& =\cosh \tilde{a}+\cosh \widetilde{c} \cosh \tilde{b}, \\
& =\cosh \tilde{a}+\cosh \widetilde{c} \cosh \tilde{b} .
\end{aligned}
$$

Comparing these two expressions for $<\widetilde{a} \times \widetilde{c}, \widetilde{a} \times \tilde{b}>$ gives the desired result. 
In the same way, we obtain dual Lorentzian cosine rule $\mathrm{I}$ for $\widetilde{\beta}$ and $\tilde{\gamma}$ as follows:

$$
\begin{aligned}
\cosh \widetilde{\beta} & =\frac{\cosh \tilde{a} \cosh \tilde{c}+\cosh \tilde{b}}{-\sinh \tilde{a} \sinh \widetilde{c}}, \\
\cosh \tilde{\gamma} & =\frac{\cosh \tilde{a} \cosh \tilde{b}+\cosh \widetilde{c}}{-\sinh \tilde{a} \sinh \widetilde{b}} .
\end{aligned}
$$

Using the equations (1) and (2) we obtain the following corollary:

Corollary 3.2. The real and dual parts of the formulas (7), (8) and (9) are given by

$$
\begin{array}{ll}
\cosh \alpha=\frac{\cosh b \cosh c+\cosh a}{-\sinh b \sinh c}, & \sinh \alpha=\frac{-\sinh a}{\alpha^{*} \sinh b \sinh c}\left(b^{*} \cosh \gamma+c^{*} \cosh \beta+a^{*}\right) ; \\
\cosh \beta=\frac{\cosh a \cosh c+\cosh b}{-\sinh a \sinh c}, & \sinh \beta=\frac{-\sinh b}{\beta^{*} \sinh a \sinh c}\left(a^{*} \cosh \gamma+c^{*} \cosh \alpha+b^{*}\right) ; \\
\cosh \gamma=\frac{\cosh a \cosh b+\cosh c}{-\sinh a \sinh b}, & \sinh \beta=\frac{-\sinh c}{\gamma^{*} \sinh a \sinh b}\left(a^{*} \cosh \beta+b^{*} \cosh \alpha+c^{*}\right),
\end{array}
$$

respectively.

In corollary 3.2, the real parts give the cosine rule $I$ for a real Lorentzian spherical triangle.

Lemma 3.3. (The Dual Lorentzian Sine Rule) Let $\widetilde{A} \widetilde{B} \widetilde{C}$ be a Lorentzian pure triangle on the dual Lorentzian unit sphere $\widetilde{S}_{1}^{2}$. Then the dual Lorentzian sine rule is given by

$$
\frac{\sinh \widetilde{\alpha}}{\sinh \widetilde{a}}=\frac{\sinh \widetilde{\beta}}{\sinh \widetilde{b}}=\frac{\sinh \tilde{\gamma}}{\sinh \widetilde{c}} .
$$

Proof: We calculate $(\tilde{c} \times \tilde{a}) \times(\tilde{a} \times \tilde{b})$ in two ways. By using the formula (6), we get

$$
\begin{aligned}
(\tilde{c} \times \tilde{a}) \times(\tilde{a} \times \tilde{b})= & \left(\tilde{n}_{b} \sinh \tilde{b}\right) \times\left(\tilde{n}_{c} \sinh \widetilde{c}\right) \\
& =\left(\tilde{n}_{b} \times \tilde{n}_{c}\right) \sinh \tilde{b} \sinh \tilde{c} \\
& =\widetilde{a} \sinh \tilde{\alpha} \sinh \tilde{b} \sinh \tilde{c} .
\end{aligned}
$$

On the other hand, by Lemma 2.1, we get

$$
\begin{aligned}
& (\widetilde{c} \times \widetilde{a}) \times(\widetilde{a} \times \widetilde{b})=-<\widetilde{c}, \widetilde{a} \times \tilde{b}>\tilde{a}+<\widetilde{a}, \widetilde{a} \times \tilde{b}>\widetilde{c} \\
& =-(<\tilde{c}, \tilde{a} \times \tilde{b}>\tilde{a}) \\
& =-(-\operatorname{det}(\widetilde{a}, \tilde{b}, \tilde{c}) \tilde{a}) \\
& =\operatorname{det}(\widetilde{a}, \widetilde{b}, \widetilde{c}) \widetilde{a} \text {. }
\end{aligned}
$$

Comparing these two expressions for $(\widetilde{c} \times \tilde{a}) \times(\widetilde{a} \times \widetilde{b})$, we have

Therefore

$$
\sinh \tilde{\alpha} \sinh \tilde{b} \sinh \widetilde{c}=\operatorname{det}(\widetilde{a}, \tilde{b}, \widetilde{c}) .
$$




$$
\frac{\sinh \widetilde{\alpha}}{\sinh \widetilde{a}}=\frac{\operatorname{det}(\widetilde{a}, \widetilde{b}, \widetilde{c})}{\sinh \widetilde{a} \sinh \widetilde{b} \sinh \widetilde{c}} .
$$

Similarly, we obtain the same formulas for $\widetilde{\beta}$ and $\tilde{\gamma}$ :

$$
\begin{aligned}
& \frac{\sinh \widetilde{\beta}}{\sinh \widetilde{b}}=\frac{\operatorname{det}(\tilde{a}, \tilde{b}, \widetilde{c})}{\sinh \widetilde{a} \sinh \widetilde{b} \sinh \widetilde{c}} \\
& \frac{\sinh \widetilde{\gamma}}{\sinh \widetilde{c}}=\frac{\operatorname{det}(\widetilde{a}, \widetilde{b}, \widetilde{c})}{\sinh \widetilde{a} \sinh \widetilde{b} \sinh \widetilde{c}} .
\end{aligned}
$$

Formulas (11), (12), (13) complete the proof.

Using the equation (1) we obtain the following corollary:

Corollary 3.4. The real and dual part of the formula (10) are given by

$$
\begin{gathered}
\frac{\sinh \alpha}{\sinh a}=\frac{\sinh \beta}{\sinh b}=\frac{\sinh \gamma}{\sinh c}, \\
\alpha^{*} \frac{\cosh \alpha}{\sinh a}-a^{*} \operatorname{coth} a \frac{\sinh \alpha}{\sinh a}=\beta^{*} \frac{\cosh \beta}{\sinh b}-b^{*} \operatorname{coth} b \frac{\sinh \beta}{\sinh b}=\gamma^{*} \frac{\cosh \gamma}{\sinh c}-c^{*} \operatorname{coth} c \frac{\sinh \gamma}{\sinh c},
\end{gathered}
$$
respectively.

The formula (14) gives sine rule for a real Lorentzian spherical triangle.

Lemma 3.5 (The Dual Lorentzian Cosine Rule II) : Let $\widetilde{A} \widetilde{B} \widetilde{C}$ be a Lorentzian pure triangle on the dual Lorentzian unit sphere $\widetilde{S}_{1}^{2}$. Then the dual Lorentzian cosine rule II is given by

$$
\cosh \widetilde{c}=\frac{\cosh \widetilde{\alpha} \cosh \widetilde{\beta}+\cosh \widetilde{\gamma}}{\sinh \widetilde{\alpha} \sinh \widetilde{\beta}}
$$

Proof: For brevity, let $\widetilde{A}, \widetilde{B}$ and $\widetilde{C}$ be $\cosh \widetilde{a}, \cosh \widetilde{b}$ and $\cosh \widetilde{c}$, respectively. Then the dual Lorentzian cosine rule I yields

$$
\begin{aligned}
\cosh \tilde{\gamma}= & \frac{\widetilde{A} \widetilde{B}+\widetilde{C}}{-\left(\widetilde{A}^{2}-1\right)^{\frac{1}{2}}\left(\widetilde{B}^{2}-1\right)^{\frac{1}{2}}} \\
\cosh \widetilde{\beta}= & \frac{\widetilde{A} \widetilde{C}+\widetilde{B}}{-\left(\widetilde{A}^{2}-1\right)^{\frac{1}{2}}\left(\widetilde{C}^{2}-1\right)^{\frac{1}{2}}} \\
\cosh \widetilde{\alpha}= & \frac{\widetilde{B} \widetilde{C}+\widetilde{A}}{-\left(\widetilde{B}^{2}-1\right)^{\frac{1}{2}}\left(\widetilde{C}^{2}-1\right)^{\frac{1}{2}}} .
\end{aligned}
$$

On the other hand, since $\cosh ^{2} \tilde{\alpha}-\sinh ^{2} \tilde{\alpha}=1$, it follows that

$$
\sinh ^{2} \tilde{\alpha}=\frac{\widetilde{D}}{\left(\widetilde{B}^{2}-1\right)\left(\widetilde{C}^{2}-1\right)},
$$


where $\widetilde{D}=-1+2 \widetilde{A} \widetilde{B} \widetilde{C}+\widetilde{A}^{2}+\widetilde{B}^{2}+\widetilde{C}^{2}$. We note that $\widetilde{D}$ is positive and symmetric in $\widetilde{A}, \widetilde{B}$ and $\widetilde{C}$. Then we obtain

$$
\begin{aligned}
& \sinh \widetilde{\alpha}=\frac{\sqrt{\widetilde{D}}}{\left(\widetilde{B}^{2}-1\right)^{\frac{1}{2}}\left(\widetilde{C}^{2}-1\right)^{\frac{1}{2}}}, \\
& \sinh \widetilde{\beta}=\frac{\sqrt{\widetilde{D}}}{\left(\widetilde{B}^{2}-1\right)^{\frac{1}{2}}\left(\widetilde{C}^{2}-1\right)^{\frac{1}{2}}}, \\
& \sinh \widetilde{\gamma}=\frac{\sqrt{\widetilde{D}}}{\left(\widetilde{A}^{2}-1\right)^{\frac{1}{2}}\left(\widetilde{B}^{2}-1\right)^{\frac{1}{2}}} .
\end{aligned}
$$

If we write the formulas (16)-(20) in the right side of the formula (15), then the equality is satisfied:

$$
\begin{aligned}
\frac{\cosh \widetilde{\alpha} \cosh \widetilde{\beta}+\cosh \widetilde{\gamma}}{\sinh \widetilde{\alpha} \sinh \widetilde{\beta}} & =\frac{(\widetilde{B} \widetilde{C}+\widetilde{A})(\widetilde{A} \widetilde{C}+\widetilde{B})+(\widetilde{A} \widetilde{B}+\widetilde{C})\left(\widetilde{C}^{2}-1\right)}{\left(\widetilde{B}^{2}-1\right)^{\frac{1}{2}}\left(\widetilde{A}^{2}-1\right)^{\frac{1}{2}}\left(\widetilde{C}^{2}-1\right)} \\
& =\frac{\left(\widetilde{A}^{2}-1\right)^{\frac{1}{2}}\left(\widetilde{B}^{2}-1\right)^{\frac{1}{2}}\left(\widetilde{C}^{2}-1\right)}{\widetilde{D}} \\
& =\frac{\widetilde{C}\left(-1+2 \widetilde{A} \widetilde{C}^{2}+\widetilde{B}^{2} \widetilde{C}+\widetilde{A}^{2} \widetilde{C}+\widetilde{A} \widetilde{B}+\widetilde{A} \widetilde{B} \widetilde{C}^{2}-\widetilde{A} \widetilde{B}+\widetilde{C}^{2}+\widetilde{D}\right.}{\widetilde{D}} \\
& =\widetilde{C} \\
& =\cosh \widetilde{c} .
\end{aligned}
$$

Similarly, we can give the similar formulas for $\cosh \tilde{b}$ and $\cosh \tilde{a}$ as follows:

$$
\begin{aligned}
\cosh \widetilde{b} & =\frac{\cosh \tilde{\gamma} \cosh \widetilde{\alpha}+\cosh \widetilde{\beta}}{\sinh \widetilde{\alpha} \sinh \tilde{\gamma}} \\
\cosh \tilde{a} & =\frac{\cosh \widetilde{\beta} \cosh \tilde{\gamma}+\cosh \widetilde{\alpha}}{\sinh \widetilde{\beta} \sinh \tilde{\gamma}}
\end{aligned}
$$

Using the equations (1) and (2) we obtain the following corollary:

Corollary 3.6. The real and dual parts of the formulas (15), (22) and (23) are given by 
$\cosh c=\frac{\cosh \alpha \cosh \beta+\cosh \gamma}{\sinh \alpha \sinh \beta}, \quad \sinh c=\frac{-\sinh \gamma}{c^{*} \sinh \alpha \sinh \beta}\left(\beta^{*} \cosh a+\alpha^{*} \cosh b-\gamma^{*}\right)$ $\cosh b=\frac{\cosh \gamma \cosh \alpha+\cosh \beta}{\sinh \gamma \sinh \alpha}, \quad \sinh b=\frac{-\sinh \beta}{b^{*} \sinh \alpha \sinh \gamma}\left(\alpha^{*} \cosh c+\gamma^{*} \cosh a-\beta^{*}\right)$ $\cosh a=\frac{\cosh \beta \cosh \gamma+\cosh \alpha}{\sinh \beta \sinh \gamma}, \quad \sinh a=\frac{-\sinh \alpha}{a^{*} \sinh \beta \sinh \gamma}\left(\gamma^{*} \cosh b+\beta^{*} \cosh c-\alpha^{*}\right)$, respectively.

In Corollary 3.6, the real parts give the cosine rule II for a real Lorentzian spherical triangle.

\section{REFERENCES}

1. K. Akutagawa; S. Nishikawa, The Gauss Map and Spacelike Surface with Prescribed Mean Curvature in Minkowski 3-Space, Tohoku Math. J., 42, 68-82, 1990.

2. A. F. Beardon, The Geometry of Discrete Groups, Springer-Verlag, New-York Berlin, 1983.

3. G. S.Birman; K. Nomizo, Trigonometry in Lorentzian Geometry, Ann. Math. Mont. 91, 543-549 1984.

4. I. N. Bronstein; K. A. Semendjajew; G. Musiol and H. Mühlig, Tashenbuch der Mathematik, Verlag Harri Deutsch, Frankfurt, 1995.

5. B. Rosenfeld, Geometry of Lie Groups, Kluwer Academic Publishers, Dordrecht Boston London, 1997.

6. E. Study, Geometrie der Dynamen, Leipzig, 1903.

7. H. H.Uğurlu; A. Çalişkan, The Study Mapping for Directed Space-Like and TimeLike Lines in Minkowski 3-Space $R_{1}^{3}$, Mathematical and Computational Applications, 1, 142-148, 1994.

8. H. H. Uğurlu; H. Gundogan, The Cosine Hyperbolic and Sine Hyperbolic Rules for Dual Hyperbolic Spherical Trigonometry, Mathematical and Computation Applications, 5, 185-190, 2000.

9. G. R. Veldkamp, On the Use of Dual Numbers, Vectors and Matrices in Instantaneous, Spatial Kinematics, Mechanism and Machine Theory, 11, 141-156, 1975. 